\title{
Dynamic Analysis of a Stochastic SEQIR Model and Application in the COVID-19 Pandemic
}

\author{
Shijie Liu and Maoxing Liu (D) \\ Department of Mathematics, North University of China, Taiyuan, Shanxi, China \\ Correspondence should be addressed to Maoxing Liu; liumxsx@gmail.com
}

Received 17 June 2021; Accepted 18 November 2021; Published 8 December 2021

Academic Editor: Li Li

Copyright (@ 2021 Shijie Liu and Maoxing Liu. This is an open access article distributed under the Creative Commons Attribution License, which permits unrestricted use, distribution, and reproduction in any medium, provided the original work is properly cited.

In this study, a deterministic SEQIR model with standard incidence and the corresponding stochastic epidemic model are explored. In the deterministic model, the reproduction number is given, and the local asymptotic stability of the equilibria is proved. When the reproduction number is less than unity, the disease-free equilibrium is locally asymptotically stable, whereas the endemic equilibrium is locally asymptotically stable in the case of a reproduction number greater than unity. A stochastic expansion based on a deterministic model is studied to explore the uncertainty of the spread of infectious diseases. Using the Lyapunov function method, the existence and uniqueness of a global positive solution are considered. Then, the extinction conditions of the epidemic and its asymptotic property around the endemic equilibrium are obtained. To demonstrate the application of this model, a case study based on COVID-19 epidemic data from France, Italy, and the UK is presented, together with numerical simulations using given parameters.

\section{Introduction}

At the end of 2019, COVID-19 was reported in Wuhan, China [1], setting the beginning of a global epidemic. Now, there are more than 200 countries trapped in the epidemic disaster, which has seriously affected the development of the society. It was found that COVID-19 was caused by the SARS-CoV-2 coronavirus [2, 3]. This coronavirus mainly spreads in three ways: (i) direct transmission, which refers to the infection caused by patient's sneezing, coughing, and talking droplets, and the direct inhalation of exhaled gas at a short distance; (ii) aerosol transmission, which refers to droplet mixing in the air to form an aerosol that can lead to infection after inhalation; (iii) contact transmission, which refers to the droplet deposited on the surface of an object, contact with contaminated hands, and then contact with the oral cavity, nasal cavity, eyes, and other mucous membranes, resulting in infection.

In the absence of a specific therapeutic method, governments can only do their best to hinder the spread of the epidemic through measures such as population lockdown, reasonable allocation of medical resources, and calls for public health (mask wearing, avoidance of places with many people, meetings with reduced attendance, etc.) $[4,5]$. These measures can effectively prevent epidemics and delay the arrival of epidemic peaks [6]. For now, through the aforementioned measures and vaccine production, the epidemic situation has been controlled in many countries; however, there are still some countries with severe epidemic situations.

Mathematical modeling is a suitable approach to describe the spread of diseases and predict their epidemic trend [7]. Based on the spread characteristics of diseases, many epidemic models, e.g., SIR, SEIR, and SIQR [8-12], were proposed. Using mathematical models to describe infectious diseases can help to better understand the spread trends of diseases and propose reasonable control methods and suggestions. Novel coronavirus pneumonia models were reported by scholars who have been involved in this task since the outbreak of the epidemic. Tang et al. constructed an SEIR model in [13]; their results indicate that the number of infected people and the propagation of infectious diseases 
can be reduced when governments take certain measures. In [14], the author predicted that the spread of infectious disease would be costly. After that, the governments of the UK and USA established strict movement restrictions. Many models, related suggestions, and opinions on COVID-19 have been provided [15-21].

Although there are many mathematical models, few considered the inherent uncertainties in these models. Manski and Molinari [22] showed that there is an uncertainty in the epidemic transmission process. Therefore, assuming that stochastic disturbance applied to a model is suitable, some stochastic epidemic models were proposed $[18,23-26]$. In [18], the authors studied an SLIRD with the deterministic and stochastic models to analyze actual COVID-19-infected cases in Spain. In the numerical simulations, they fitted data from Spain and obtained the daily basic reproduction number. Thus, they predicted the epidemic situation for the next few months. In [23], by extending the SIR epidemiological model, the authors established a random propagation model for additional modeling of whether an individual is far from a crowded area. The results showed that the propagation of the epidemic in Japan would gradually slow down by reducing the time spent in crowded zones. Anwarud et al. analyzed a stochastic SIQ model and obtained sufficient conditions for disease extinction, disease existence, and stationary distribution in [24]. The numerical simulations were divided into two parts: fitting data from Khyber Pakhtunkhwa, Pakistan, and verification of the theoretical results for the given parameters. In [25], the authors studied an SEQIR model with Markovian switching, established a random threshold of disease extinction and persistence, and used the data from Indian states to confirm their conclusions. In [26], an epidemic system was considered through an additive fractional white noise. It was indicated that the epidemic under fractional random settings may be more suitable for modeling than deterministic modeling. Based on the above studies, an SEQIR model with standard incidence is analyzed in this study, together with deterministic and stochastic models. A case study of COVID-19 epidemic data from France, Italy, and the UK is presented, and numerical simulations using given parameters are reported.

The remainder of this paper is organized as follows. In the next section, deterministic and stochastic SEQIR models with standard incidence are described. The dynamic analysis of the deterministic model is presented in Section 3, and an analysis of the stochastic system is presented in Section 4. Numerical simulations and application to the COVID-19 epidemic are described in Section 5. The paper ends with a discussion in Section 6.

\section{Model Formulation}

The entire population is divided into five classes according to its states, namely, susceptible $S(t)$, exposed $E(t)$, quarantined $Q(t)$, hospitalized infected $I(t)$, and recovered $R(t)$. Then, $N(t)=S(t)+E(t)+Q(t)+I(t)+R(t)$. In [19],
Mandal et al. constructed an SEQIR model with optimized control and provided advice to Indian cities. Based on their work, Brahim et al. considered a model with Markovian switching and generalized incidence in [25]. Interestingly, they considered that people who are exposed and asymptomatic are infectious. The standard incidence rate is more suitable for infectious disease models with a large population. Based on these results, we consider an SEQIR model with standard incidence as follows:

$$
\begin{aligned}
& \frac{\mathrm{d} S(t)}{\mathrm{d} t}=A-\frac{\beta S(t) E(t)}{N(t)}+b_{1} Q(t)-d S(t), \\
& \frac{\mathrm{d} E(t)}{\mathrm{d} t}=\frac{\beta S(t) E(t)}{N(t)}-\left(b_{2}+\alpha+d\right) E(t), \\
& \frac{\mathrm{d} Q(t)}{\mathrm{d} t}=b_{2} E(t)-\left(b_{1}+c+d\right) Q(t), \\
& \frac{\mathrm{d} I(t)}{\mathrm{d} t}=\alpha E(t)+c Q(t)-(\eta+d+\delta) I(t), \\
& \frac{\mathrm{d} R(t)}{\mathrm{d} t}=\eta I(t)-d R(t) .
\end{aligned}
$$

The constant input of the susceptible population is $A ; \beta$ represents the transmission rate of the disease; $\alpha$ is the rate of the exposed class removed from the infected class; $b_{2}$ is the portion of the exposed class moving to the quarantined class; $b_{1}$ and $c$ indicate the rate at which the quarantined people become susceptible and infected types, respectively; the recovery rate of hospitalized infected people is $\eta$; $d$ is the natural mortality rate; and $\delta$ is the diseased death rate.

A popular technique to incorporate stochasticity into a deterministic model is parametric perturbation. Following this method, the transmission rate, $\beta$, is replaced by $\beta \longrightarrow \beta+\sigma \mathrm{d} B(t)$. Therefore, we have a stochastic model defined as follows:

$$
\begin{aligned}
& \mathrm{d} S(t)=\left(A-\frac{\beta S(t) E(t)}{N(t)}+b_{1} Q(t)-\mathrm{d} S(t)\right) \mathrm{d} t-\frac{\sigma S(t) E(t)}{N(t)} \mathrm{d} B(t), \\
& \mathrm{d} E(t)=\left(\frac{\beta S(t) E(t)}{N(t)}-\left(b_{2}+\alpha+d\right) E(t)\right) \mathrm{d} t+\frac{\sigma S(t) E(t)}{N(t)} \mathrm{d} B(t), \\
& \mathrm{d} Q(t)=\left(b_{2} E(t)-\left(b_{1}+c+d\right) Q(t)\right) \mathrm{d} t \\
& \mathrm{~d} I(t)=(\alpha E(t)+c Q(t)-(\eta+d+\delta) I(t)) \mathrm{d} t \\
& \mathrm{~d} R(t)=(\eta I(t)-\mathrm{d} R(t)) \mathrm{d} t
\end{aligned}
$$

Note that $B(t)$ is a standard independent Brownian motion, and the intensity of the white noise is $\sigma$. An initial condition should be satisfied when analyzing systems (1) and (2):

$$
S(0) \geq 0, E(0) \geq 0, Q(0) \geq 0, I(0) \geq 0, R(0) \geq 0 .
$$


In the following analysis, we declare that the standard Brownian motion in the system is defined on a complete probability space $(\Omega, \mathscr{F}, P)$ with a filtration $\left\{\mathscr{F}_{t}\right\}_{t \in R_{+}}$satisfying the usual conditions (i.e., it is right continuous and increasing while $\mathscr{F}_{0}$ contains all $P$-null sets).

\section{Analysis of the Deterministic Model}

In this section, we analyze system (1) without stochastic perturbation. In this part, some of the basic dynamics are analyzed, including the basic reproduction number and stability of the equilibria.

3.1. The Basic Reproduction Number. An important parameter in infectious diseases is the basic reproduction number which can determine the outbreak or extinction of the disease. Therefore, we give it by the next-generation matrix method [27]. Because the infection classes involve $E$, $Q$, and $I$ classes, we consider the three classes:

$$
\begin{aligned}
& \frac{\mathrm{d} E(t)}{\mathrm{d} t}=\frac{\beta S(t) E(t)}{N(t)}-\left(b_{2}+\alpha+d\right) E(t), \\
& \frac{\mathrm{d} Q(t)}{\mathrm{d} t}=b_{2} E(t)-\left(b_{1}+c+d\right) Q(t), \\
& \frac{\mathrm{d} I(t)}{\mathrm{d} t}=\alpha E(t)+c Q(t)-(\eta+d+\delta) I(t) .
\end{aligned}
$$

Infection subsystem (4) has transmission matrix $F$ and transition matrix $V$, where

$$
\begin{aligned}
F & =\left[\begin{array}{lll}
\beta & 0 & 0 \\
0 & 0 & 0 \\
0 & 0 & 0
\end{array}\right], \\
V & =\left[\begin{array}{ccc}
b_{2}+\alpha+d & 0 & 0 \\
-b_{2} & b_{1}+c+d & 0 \\
-\alpha & -c & \eta+d+\delta
\end{array}\right] .
\end{aligned}
$$

So, according to this method, we can get that the spectral radius of $F V^{-1}$ is $R_{0}=\beta /\left(b_{2}+\alpha+d\right)$.

3.2. The Equilibria. System (1) has two equilibria, the disease-free equilibrium $E_{0}=(A / d, 0,0,0,0)$ and the endemic equilibrium $E_{*}=\left(S^{*}, E^{*}, Q^{*}, I^{*}, R^{*}\right)$, respectively, where

$$
\begin{aligned}
S^{*} & =\frac{\left(b_{2}+\alpha+d\right) N^{*}}{\beta}, \\
E^{*} & =\frac{\left(-N^{*} d\left(\alpha+b_{2}+d\right)+A \beta\right)\left(b_{1}+c+d\right)}{\beta\left((\alpha+d)\left(b_{1}+c+d\right)+b_{2}(c+d)\right)}, \\
Q^{*} & =\frac{\left[A \beta-N^{*} d\left(b_{2}+\alpha+d\right)\right] b_{2}}{\left(\left(b_{1}+b_{2}+\alpha+c+d\right) d+\left(b_{1}+c\right) \alpha+b_{2} c\right) \beta}, \\
I^{*} & =\frac{\alpha\left(b_{1}+c+d\right)+b_{2} c}{b_{2}(\eta+d+\delta)} Q^{*}, \\
R^{*} & =\frac{\eta}{d} I^{*} .
\end{aligned}
$$

Here, $N^{*}=S^{*}+E^{*}+Q^{*}+I^{*}+R^{*}$. It is worth noting that the endemic equilibrium $E_{*}$ exists when $R_{0}>1$.

3.3. The Stability of Two Equilibria. In this part, we study the local asymptotic stability of two equilibria.

Theorem 1. The disease-free equilibrium $E_{0}$ is locally asymptotically stable if $R_{0}<1$.

Proof. The Jacobian matrix at the disease-free equilibrium of system (1) is given by

$$
\left.J\right|_{E_{0}}=\left[\begin{array}{ccccc}
-d & -\beta & b_{1} & 0 & 0 \\
0 & \beta-\left(b_{2}+\alpha+d\right) & 0 & 0 & 0 \\
0 & b_{2} & -\left(b_{1}+c+d\right) & 0 & 0 \\
0 & \alpha & c & -(\eta+d+\delta) & 0 \\
0 & 0 & 0 & \eta & -d
\end{array}\right] .
$$

The characteristic equation of system (1) at its diseasefree equilibrium is given by

$$
\begin{aligned}
& (\lambda+d)\left(\lambda+\left(b_{2}+\alpha+d\right)\left(1-R_{0}\right)\right)\left(\lambda+b_{1}+c+d\right) \\
& (\lambda+\eta+d+\delta)(\lambda+d)=0 .
\end{aligned}
$$

It is easy to see that the characteristic values of the Jacobian matrix are negative if and only if $R_{0}<1$. Hence, the disease-free equilibrium of system (1) is locally asymptotically stable if $R_{0}<1$.

Theorem 2. The endemic equilibrium $E_{*}$ is locally asymptotically stable for $R_{0}>1$.

Proof. The Jacobian matrix at the endemic equilibrium of system (1) is given by 


$$
\left.J\right|_{E_{*}}=\left[\begin{array}{ccccc}
-\frac{\beta E^{*}}{N^{*}}-d & -\frac{\beta S^{*}}{N^{*}} & b_{1} & 0 & 0 \\
\frac{\beta E^{*}}{N^{*}} & \frac{\beta S^{*}}{N^{*}}-\left(b_{2}+\alpha+d\right) & 0 & 0 & 0 \\
0 & b_{2} & -\left(b_{1}+c+d\right) & 0 & 0 \\
0 & \alpha & c & -(\eta+d+\delta) & 0 \\
0 & 0 & 0 & \eta & -d
\end{array}\right] \text {. }
$$

The characteristic equation of system (1) at its endemic equilibrium is given by

$$
(\lambda+d)(\lambda+\eta+d+\delta)\left(\lambda^{3}+a_{1} \lambda^{2}+a_{2} \lambda+a_{3}\right)=0
$$

where $a_{1}=b_{1}+c+2 d+\left(\beta E^{*} / N^{*}\right), a_{2}=\left(\left(\beta E^{*} / N^{*}\right)+d\right)$ $\left(b_{1}+c+d\right)+\left(\beta^{2} E^{*} S^{*} /\left(N^{*}\right)^{2}\right)$, and $a_{3}=\left(\beta E^{*} / N^{*}\right)\left[b_{1}(\alpha+\right.$ $\left.d)+(c+d)\left(b_{2}+\alpha+d\right)\right]$. It is clear that the characteristic polynomial has two negative eigenvalues, and we study the last cubic polynomial. By calculation, we can verify $a_{1}, a_{2}, a_{3}>0$ and $a_{1} a_{2}-a_{3}>0$. Hence, according to the Routh-Hurwitz criterion, we can know that the endemic equilibrium of system (1) is locally asymptotically stable for $R_{0}>1$.

\section{Analysis of the Stochastic Model}

Theorem 3. For any initial values $(S(0), E(0), Q(0), I(0), R(0)) \in R_{+}^{5}$, there exists a unique global solution $(S(t), E(t), Q(t), I(t), R(t)) \in R_{+}^{5}$ of system (2) for all $t \geq 0$, and the solution will remain in $R_{+}^{5}$ with probability 1, i.e., $(S(t), E(t), Q(t), I(t), R(t)) \in R_{+}^{5}$ for all $t \geq 0$ almost surely.

Proof. Since the coefficients of system (2) are locally Lipschitz continuous on $R_{+}$, for any initial value
$(S(0), E(0), Q(0), I(0), R(0)) \in R_{+}^{5}$, there exists a unique local solution $(S(t), E(t), Q(t), I(t), R(t))$ on $\left[0, \tau_{e}\right)$, where $\tau_{e}$ represents the explosion time [28]. If we can prove $\tau_{e}=\infty$ a.s, then the solution is global. To this end, let $k_{0} \geq 1$ be sufficiently large such that $S(0), E(0), Q(0), I(0)$, and $R(0)$ all lie in the interval $\left[1 / k_{0}, k_{0}\right]$. For each integer $k \geq k_{0}$, we can define the stopping time [28] through $\tau_{k}=\inf \left\{t \in\left[0, \tau_{e}\right): S \quad(t) \notin(1 / k, k)\right.$ or $E(t) \notin(1 / k, k)$ or $Q(t) \notin(1 / k, k)$ or $I(t) \notin(1 / k, k)$ or $R(t) \notin(1 / k, k)\}$, where, throughout this paper, we set inf $\varnothing=\infty$ (as usual $\varnothing$ represents the empty set). Obviously, $\tau_{k}$ is increasing as $k \longrightarrow \infty$. Let $\tau_{\infty}=\lim _{k \longrightarrow \infty} \tau_{k}$, whence $\tau_{\infty} \leq \tau_{e}$ a.s. If we can show $\tau_{\infty}=+\infty$, then $\tau_{e}=+\infty$ and $(S(t), E(t), Q(t), I(t), R(t)) \in R_{+}^{5}$ a.s for all $t \geq 0$. That is to say, to complete the proof, all we need is to show that $\tau_{\infty}=$ $+\infty$ a.s. If this assertion is false, then there exists a pair of constants $T>0$ and $\varepsilon_{1} \in(0,1)$ such that $P\left\{\tau_{\infty} \leq T\right\}>\varepsilon_{1}$. There is an integer $k_{1} \geq k_{0}$ such that $P\left\{\tau_{k} \leq T\right\} \geq \varepsilon_{1}$ for all $k \geq$ $k_{1}$. Define a Lyapunov function $V: R_{+} \longrightarrow R_{+}$by $V(S, E, Q$, $I, R)=(S-1-\ln S)+(E-1-\ln E)+(Q-1-\ln Q)+(I$ $-1-\ln I)+(R-1-\ln R)$.

Since $u-1-\ln u \geq 0$ for any $u>0$, the function $V$ is nonnegative. Making use of It $\widehat{o}$ 's formula (see [28]) to $V$, we get

$$
\begin{aligned}
L V= & \left(1-\frac{1}{S}\right)\left(A-\frac{\beta S E}{N}+b_{1} Q-\mathrm{d} S\right)+\frac{\sigma^{2} E^{2}}{2 N^{2}}+\left(1-\frac{1}{E}\right)\left(\frac{\beta S E}{N}-\left(b_{2}+\alpha+d\right) E\right)+\frac{\sigma^{2} S^{2}}{2 N^{2}} \\
& +\left(1-\frac{1}{Q}\right)\left(b_{2} E-\left(b_{1}+c+d\right) Q\right)+\left(1-\frac{1}{I}\right)(\alpha E+c Q-(\eta+d+\delta) I)+\left(1-\frac{1}{R}\right)(\eta I-\mathrm{d} R) \\
= & A-\frac{\beta S E}{N}+b_{1} Q-\mathrm{d} S-\frac{A}{S}+\frac{\beta E}{N}-\frac{b_{1} Q}{S}+d+\frac{\sigma^{2} E^{2}}{2 N^{2}}+\frac{\beta S E}{N}-\left(b_{2}+\alpha+d\right) E-\frac{\beta S}{N} \\
& +\left(b_{2}+\alpha+d\right)+\frac{\sigma^{2} S^{2}}{2 N^{2}}+b_{2} E-\left(b_{1}+c+d\right) Q-\frac{b_{2} E}{Q}+\left(b_{1}+c+d\right)+\alpha E+c Q \\
& -(\eta+d+\delta) I-\frac{\alpha E}{I}-\frac{c Q}{I}+(\eta+d+\delta)+\eta I-\mathrm{d} R-\frac{\eta I}{R}+d \\
& \leq A+\beta+\alpha+b_{1}+b_{2}+\eta+\delta+c+5 d+\sigma^{2} \triangleq K, \\
\mathrm{~d} V= & L V \mathrm{~d} t+\frac{\sigma(E-S)}{N} \mathrm{~d} B(t) \leq K \mathrm{~d} t+\frac{\sigma(E-S)}{N} \mathrm{~d} B(t),
\end{aligned}
$$


where $K$ is a positive constant. Integrating from 0 to $\tau_{k} \wedge T=$ $\min \left\{\tau_{k}, T\right\}$ and then taking the expectation on both sides, we obtain

$$
E V\left(S\left(\tau_{k} \wedge T\right), E\left(\tau_{k} \wedge T\right), Q\left(\tau_{k} \wedge T\right), I\left(\tau_{k} \wedge T\right), R\left(\tau_{k} \wedge T\right)\right) \leq V(S(0), E(0), Q(0), I(0), R(0))+K E\left(\tau_{k} \wedge T\right) .
$$

Thus, we have

$$
E V\left(S\left(\tau_{k} \wedge T\right), E\left(\tau_{k} \wedge T\right), Q\left(\tau_{k} \wedge T\right), I\left(\tau_{k} \wedge T\right), R\left(\tau_{k} \wedge T\right)\right) \leq V(S(0), E(0), Q(0), I(0), R(0))+K T .
$$

Set $\Omega_{k}=\left\{\tau_{k} \leq T\right\}$ for $k \geq k_{1}$, and we get $P\left(\Omega_{k}\right) \geq \varepsilon_{1}$. Note that, for every $\omega \in \Omega_{k}$, there exists that $S\left(\tau_{k}, \omega\right)$, $E\left(\tau_{k}, \omega\right), Q\left(\tau_{k}, \omega\right), I\left(\tau_{k}, \omega\right), R\left(\tau_{k}, \omega\right)$ equals either $k$ or $(1 / k)$.
Therefore, $V\left(S\left(\tau_{k}, \omega\right), E\left(\tau_{k}, \omega\right), Q\left(\tau_{k}, \omega\right), I\left(\tau_{k}, \omega\right), R\left(\tau_{k}, \omega\right)\right)$ is no less than either $k-1-\ln k$ or $(1 / k)-1-\ln$ $(1 / k)=(1 / k)-1+\ln k$. Therefore, we can see

$$
V\left(S\left(\tau_{k}, \omega\right), E\left(\tau_{k}, \omega\right), Q\left(\tau_{k}, \omega\right), I\left(\tau_{k}, \omega\right), R\left(\tau_{k}, \omega\right)\right) \geq(k-1-\ln k) \wedge\left(\frac{1}{k}-1+\ln k\right)
$$

Then, we get

$$
\begin{aligned}
V(S(0), E(0), Q(0), I(0), R(0))+K T & \geq E\left[1_{\Omega_{k}(\omega)} V\left(S\left(\tau_{k}, \omega\right), E\left(\tau_{k}, \omega\right), Q\left(\tau_{k}, \omega\right), I\left(\tau_{k}, \omega\right), R\left(\tau_{k}, \omega\right)\right)\right] \\
& \geq \varepsilon_{1}(k-1-\ln k) \wedge\left(\frac{1}{k}-1+\ln k\right)
\end{aligned}
$$

where $1_{\Omega_{k}(\omega)}$ is the indicator function of $\Omega_{k}$. Letting $k \longrightarrow \infty$,

$$
+\infty>V(S(0), E(0), Q(0), I(0), R(0))+K T=+\infty .
$$

is a contradiction, and thus, we can get $\tau_{\infty}=+\infty$ a.s., which implies that $(S(t), E(t), Q(t), I(t), R(t)) \in R_{+}^{5}$ for all $t \in R_{+}$. This completes the proof.

Remark 1. Define $\Gamma=\left\{(S(t), E(t), Q(t), I(t), R(t)) \in R_{+}^{5}\right.$ : $A /(d+\delta) \leq S(t)+E(t)+Q(t)+I(t)+R(t) \leq(A / d)\}$. By system (2), we can get $(\mathrm{d} / \mathrm{d} t)(S+E+Q+I+R)=$ $A-d(S+E+Q+I+R)-\delta I$. Next, we have $A-(d+\delta)$ $(S+E+Q+I+R) \leq A-d(S+E+Q+I+R)-\delta I \leq A-d$ $(S+E+Q+I+R)$. Therefore, we can obtain the solution of system (2) in the region $\Gamma$. Then, we can give some general conclusions and results.
Theorem 4. Let $(S(t), E(t), Q(t), I(t), R(t))$ be the positive solution of system (2) with the initial value $(S(0), E(0), Q(0), I(0), R(0)) \in R_{+}^{5}$. If the assumption $\left(\beta^{2} / 2 \sigma^{2}\right)<b_{2}+\alpha+d$ holds, then the disease will go to extinction exponentially with probability one, i.e.,

$$
\lim _{t \rightarrow \infty} E(t)=\lim _{t \longrightarrow \infty} Q(t)=\lim _{t \longrightarrow \infty} I(t)=\lim _{t \longrightarrow \infty} R(t)=0 \text {, a.s. }
$$

Proof. Applying Itôo's formula, we have

$$
d \ln E(t)=\left[\frac{\beta S}{N}-\left(b_{2}+\alpha+d\right)-\frac{\sigma^{2} S^{2}}{2 N^{2}}\right] \mathrm{d} t+\frac{\sigma S}{N} \mathrm{~d} B(t) .
$$

Integrating (18) from 0 to $t$, we get 


$$
\begin{aligned}
\ln E(t)-\ln E(0) & =\int_{0}^{t}\left[\frac{\beta S(u)}{N(u)}-\left(b_{2}+\alpha+d\right)-\frac{\sigma^{2} S^{2}(u)}{2 N^{2}(u)}\right] \mathrm{d} u+\int_{0}^{t} \frac{\sigma S(u)}{N(u)} \mathrm{d} B(u) \\
& =\int_{0}^{t}\left(-\frac{\sigma^{2} S^{2}(u)}{2 N^{2}(u)}+\frac{\beta S(u)}{N(u)}\right) \mathrm{d} u-\left(b_{2}+\alpha+d\right) t+M(t),
\end{aligned}
$$

where $M(t)=\int_{0}^{t}(\sigma S(u) / N(u)) \mathrm{d} B(u)$ is a continuous local martingale [28] whose quadratic variation is

$$
\langle M, M\rangle(t)=\sigma^{2} \int_{0}^{t} \frac{S^{2}(u)}{N^{2}(u)} \mathrm{d} u .
$$

Applying Theorem 7.4 in [28], p44, we can get

$$
P\left\{\sup _{0 \leq t \leq k}\left[M(t)-\frac{c}{2}\langle M, M\rangle(t)\right]>\frac{2}{c} \ln k\right\} \leq \frac{1}{k^{2}}
$$

$\omega \in \Omega$, there exists a random integer $k_{0}(\omega)$ such that, for all $k>k_{0}$,

$$
\sup _{0 \leq t \leq k}\left[M(t)-\frac{c}{2}\langle M, M\rangle(t)\right] \leq \frac{2}{c} \ln k
$$

That is to say,

$$
M(t) \leq \frac{c}{2} \sigma^{2} \int_{0}^{t} \frac{S^{2}(u)}{N^{2}(u)} \mathrm{d} u+\frac{2}{c} \ln k, \quad \text { for any } 0 \leq t \leq k .
$$

where $0<c<1$ and $k>1$ is a random integer. Using the Borel-Cantelli lemma [28], we obtain that, for almost all
Taking it to (19), we obtain

$$
\ln E(t)-\ln E(0) \leq \int_{0}^{t}\left(-\frac{1}{2}(1-c) \frac{\sigma^{2} S^{2}(u)}{N^{2}(u)}+\frac{\beta S(u)}{N(u)}\right) \mathrm{d} u-\left(b_{2}+\alpha+d\right) t+\frac{2}{c} \ln k
$$

Observing the integrand function, we can do the following:

$$
-\frac{1}{2}(1-c) \frac{\sigma^{2} S^{2}(u)}{N^{2}(u)}+\frac{\beta S(u)}{N(u)} \leq \frac{\beta^{2}}{2(1-c) \sigma^{2}} .
$$

Consequently, we get

$\ln E(t)-\ln E(0) \leq \frac{\beta^{2}}{2(1-c) \sigma^{2}} t-\left(b_{2}+\alpha+d\right) t+\frac{2}{c} \ln k$.

Therefore, for $k-1 \leq t \leq k$, one can see that

$$
\frac{\ln E(t)}{t} \leq \frac{\ln E(0)}{t}+\frac{\beta^{2}}{2(1-c) \sigma^{2}}-\left(b_{2}+\alpha+d\right)+\frac{2}{c} \frac{\ln k}{k-1} \text {. }
$$

Letting $k \longrightarrow+\infty, t \longrightarrow+\infty,(\ln E(0) / t) \longrightarrow 0$, and $(\ln k /(k-1)) \longrightarrow 0$. We can obtain

$$
\limsup _{t \rightarrow+\infty} \frac{\ln E(t)}{t} \leq \frac{\beta^{2}}{2(1-c) \sigma^{2}}-\left(b_{2}+\alpha+d\right) \text { a.s. }
$$

Letting $c \longrightarrow 0$ gives

$$
\limsup _{t \longrightarrow+\infty} \frac{\ln E(t)}{t} \leq \frac{\beta^{2}}{2 \sigma^{2}}-\left(b_{2}+\alpha+d\right) \text { a.s, }
$$

which shows that $\lim _{t \longrightarrow+\infty} E(t)=0$ a.s.
On the other hand, there exists a constant $T$ such that $E(w) \leq \varepsilon, t>T$. Applying the third equation of system (2), we get

$$
\mathrm{d} Q(t)=\left[b_{2} E-\left(b_{1}+c+d\right) Q\right] \mathrm{d} t \leq\left[b_{2} \varepsilon-\left(b_{1}+c+d\right) Q\right] \mathrm{d} t .
$$

Using the comparison theorem, we have

$$
\limsup _{t \longrightarrow+\infty} Q(t) \leq \frac{b_{2} \varepsilon}{b_{1}+c+d} \text { a.s. }
$$

Letting $\varepsilon \longrightarrow 0$, we get $\lim _{t \longrightarrow+\infty} Q(t)=0$ a.s. Similarly, in the case that $\lim _{t \longrightarrow+\infty} E(t)=0$ a.s. and $\lim _{t \longrightarrow+\infty} Q(t)=0$ a.s., we can get

$$
\begin{aligned}
& \lim _{t \longrightarrow+\infty} I(t)=0 \text { a.s., } \\
& \lim _{t \longrightarrow+\infty} R(t)=0 \text { a.s. }
\end{aligned}
$$

This completes the proof.

Theorem 5. Assume that $R_{0}>1, m_{1}=d-\left(b_{1} / 2\right)-\left(\sigma^{2}\right.$ $\left.(d+\delta)\left(b_{2}+\alpha+2 d\right) E^{*} / \beta A\right), \quad m_{2}=\left(b_{2} / 2\right)+(\alpha / 2)+d-$ $\left(b_{1} / 2\right), \quad m_{3}=(c / 2)+d-\left(b_{2} / 2\right), \quad m_{4}=(\eta / 2)+d+\delta-$ $(\alpha / 2)-(c / 2), \quad m_{5}=d-(\eta / 2), \quad M=\min \left\{m_{1}, m_{2}, m_{3}\right.$, $\left.m_{4}, m_{5}\right\}$, and $\Lambda=\left(\sigma^{2}(d+\delta)\left(b_{2}+\alpha+2 d\right) E^{*} / \beta A\right)\left(S^{*}\right)^{2}+$ $\left(A S^{*} \delta\left(b_{2}+\alpha+2 d\right) / d(d+\delta)\right)$ are all positive constants. Then, for any initial value $(S(0), E(0), Q(0)$, $I(0), R(0)) \in R_{+}^{5}$, the solution of system (2) has the property 


$$
\limsup _{t \rightarrow \infty} \frac{1}{t} E \int_{0}^{t}\left[\left(S-S^{*}\right)^{2}+\left(E-E^{*}\right)^{2}+\left(Q-Q^{*}\right)^{2}+\left(I-I^{*}\right)^{2}+\left(R-R^{*}\right)^{2}\right] \mathrm{d} u \leq \frac{\Lambda}{M}
$$

Proof. System (1) has the endemic equilibrium, and we can get

$$
\begin{aligned}
A & =\frac{\beta S^{*} E^{*}}{N^{*}}-b_{1} Q^{*}+\mathrm{d} S^{*}, \\
\frac{\beta S^{*} E^{*}}{N^{*}} & =\left(b_{2}+\alpha+d\right) E^{*}, \\
b_{2} E^{*} & =\left(b_{1}+c+d\right) Q^{*}, \\
\alpha E^{*}+c Q^{*} & =(\eta+d+\delta) I^{*}, \\
\eta I^{*} & =\mathrm{d} R^{*} .
\end{aligned}
$$

We construct a function$$
V=\frac{1}{2}\left(S-S^{*}+E-E^{*}\right)^{2}+\chi\left(E-E^{*}-E^{*} \ln \frac{E}{E^{*}}\right)+\frac{1}{2}\left(Q-Q^{*}\right)^{2}+\frac{1}{2}\left(I-I^{*}\right)^{2}+\frac{1}{2}\left(R-R^{*}\right)^{2}=V_{1}+\chi V_{2}+V_{3}+V_{4}+V_{5}
$$

where $\chi$ is a constant. By Itô's formula, we obtain

$$
L V=L V_{1}+\chi L V_{2}+L V_{3}+L V_{4}+L V_{5} \text {. }
$$

And we get

$$
\begin{aligned}
& \leq-\left(d-\frac{b_{1}}{2}\right)\left(S-S^{*}\right)^{2}-\left(b_{2}+\alpha+d-\frac{b_{1}}{2}\right)\left(E-E^{*}\right)^{2}+b_{1}\left(Q-Q^{*}\right)^{2} \\
& -\left(b_{2}+\alpha+2 d\right)\left(S-S^{*}\right)\left(E-E^{*}\right) \\
& L V_{2}=\left(1-\frac{E^{*}}{E}\right)\left(\frac{\beta S E}{N}-\left(b_{2}+\alpha+d\right) E\right)+\frac{\sigma^{2} S^{2}}{2 N^{2}} E^{*} \\
& \begin{array}{l}
\leq\left(E-E^{*}\right)\left(\frac{\beta S(d+\delta)}{A}-\frac{\beta S^{*}(d+\delta)}{A}+\frac{\beta S^{*} \delta}{A}\right)+\frac{\sigma^{2} S^{2}(d+\delta)^{2}}{2 A^{2}} E^{*} \\
\leq \frac{\beta(d+\delta)}{A}\left(S-S^{*}\right)\left(E-E^{*}\right)+\frac{\sigma^{2}(d+\delta)^{2}\left(S-S^{*}+S^{*}\right)^{2}}{2 A^{2}} E^{*}+\frac{\beta S^{*} \delta}{d}
\end{array} \\
& \begin{array}{l}
\leq\left(E-E^{*}\right)\left(\frac{\beta S(d+\delta)}{A}-\frac{\beta S^{*}(d+\delta)}{A}+\frac{\beta S^{*} \delta}{A}\right)+\frac{\sigma^{2} S^{2}(d+\delta)^{2}}{2 A^{2}} E^{*} \\
\leq \frac{\beta(d+\delta)}{A}\left(S-S^{*}\right)\left(E-E^{*}\right)+\frac{\sigma^{2}(d+\delta)^{2}\left(S-S^{*}+S^{*}\right)^{2}}{2 A^{2}} E^{*}+\frac{\beta S^{*} \delta}{d}
\end{array} \\
& \leq \frac{\beta(d+\delta)}{A}\left(S-S^{*}\right)\left(E-E^{*}\right)+\frac{\sigma^{2}(d+\delta)^{2}\left(S-S^{*}\right)^{2}}{A^{2}} E^{*} \\
& +\frac{\sigma^{2}(d+\delta)^{2}\left(S^{*}\right)^{2}}{A^{2}} E^{*}+\frac{\beta S^{*} \delta}{d} \\
& L V_{1}=\left(S-S^{*}+E-E^{*}\right)\left(A-d S+b_{1} Q-\left(b_{2}+\alpha+d\right) E\right) \\
& =-d\left(S-S^{*}\right)^{2}-\left(b_{2}+\alpha+d\right)\left(E-E^{*}\right)^{2}-\left(b_{2}+\alpha+2 d\right)\left(S-S^{*}\right)\left(E-E^{*}\right) \\
& +b_{1}\left(S-S^{*}\right)\left(Q-Q^{*}\right)+b_{1}\left(E-E^{*}\right)\left(Q-Q^{*}\right)
\end{aligned}
$$




$$
\begin{aligned}
L V_{3} & =\left(Q-Q^{*}\right)\left(b_{2} E-\left(b_{1}+c+d\right) Q\right) \\
& =b_{2}\left(E-E^{*}\right)\left(Q-Q^{*}\right)-\left(b_{1}+c+d\right)\left(Q-Q^{*}\right)^{2} \\
& \leq-\left(b_{1}+c+d-\frac{b_{2}}{2}\right)\left(Q-Q^{*}\right)^{2}+\frac{b_{2}}{2}\left(E-E^{*}\right)^{2}, \\
L V_{4} & =\left(I-I^{*}\right)(\alpha E+c Q-(\eta+d+\delta) I) \\
& =\alpha\left(E-E^{*}\right)\left(I-I^{*}\right)+c\left(Q-Q^{*}\right)\left(I-I^{*}\right)-(\eta+d+\delta)\left(I-I^{*}\right)^{2} \\
& \leq-\left(\eta+d+\delta-\frac{\alpha}{2}-\frac{c}{2}\right)\left(I-I^{*}\right)^{2}+\frac{\alpha}{2}\left(E-E^{*}\right)^{2}+\frac{c}{2}\left(Q-Q^{*}\right)^{2}, \\
L V_{5} & =\left(R-R^{*}\right)(\eta I-\mathrm{d} R) \\
& =\eta\left(I-I^{*}\right)\left(R-R^{*}\right)-d\left(R-R^{*}\right)^{2} \leq-\left(d-\frac{\eta}{2}\right)\left(R-R^{*}\right)^{2}+\frac{\eta}{2}\left(I-I^{*}\right)^{2} .
\end{aligned}
$$

Taking (37)-(41) into (36), one can obtain that

$$
\begin{aligned}
& L V \leq-\left(d-\frac{b_{1}}{2}\right)\left(S-S^{*}\right)^{2}-\left(b_{2}+\alpha+d-\frac{b_{1}}{2}\right)\left(E-E^{*}\right)^{2}+b_{1}\left(Q-Q^{*}\right)^{2} \\
& -\left(b_{2}+\alpha+2 d\right)\left(S-S^{*}\right)\left(E-E^{*}\right)+\chi\left[\frac{\beta(d+\delta)}{A}\left(S-S^{*}\right)\left(E-E^{*}\right)\right. \\
& \left.+\frac{\sigma^{2}(d+\delta)^{2}\left(S-S^{*}\right)^{2}}{A^{2}} E^{*}+\frac{\sigma^{2}(d+\delta)^{2}\left(S^{*}\right)^{2}}{A^{2}} E^{*}+\frac{\beta S^{*} \delta}{d}\right]+\frac{b_{2}}{2}\left(E-E^{*}\right)^{2} \\
& -\left(b_{1}+c+d-\frac{b_{2}}{2}\right)\left(Q-Q^{*}\right)^{2}-\left(\eta+d+\delta-\frac{\alpha}{2}-\frac{c}{2}\right)\left(I-I^{*}\right)^{2} \\
& +\frac{\alpha}{2}\left(E-E^{*}\right)^{2}+\frac{c}{2}\left(Q-Q^{*}\right)^{2}-\left(d-\frac{\eta}{2}\right)\left(R-R^{*}\right)^{2}+\frac{\eta}{2}\left(I-I^{*}\right)^{2} \\
& \leq-\left(d-\frac{b_{1}}{2}-\frac{\sigma^{2}(d+\delta)\left(b_{2}+\alpha+2 d\right) E^{*}}{\beta A}\right)\left(S-S^{*}\right)^{2}-\left(\frac{b_{2}}{2}+\frac{\alpha}{2}+d-\frac{b_{1}}{2}\right)\left(E-E^{*}\right)^{2} \\
& -\left(\frac{c}{2}+d-\frac{b_{2}}{2}\right)\left(Q-Q^{*}\right)^{2}-\left(\frac{\eta}{2}+d+\delta-\frac{\alpha}{2}-\frac{c}{2}\right)\left(I-I^{*}\right)^{2} \\
& -\left(d-\frac{\eta}{2}\right)\left(R-R^{*}\right)^{2}+\frac{\sigma^{2}(d+\delta)\left(b_{2}+\alpha+2 d\right) E^{*}}{\beta A}\left(S^{*}\right)^{2}+\frac{A S^{*} \delta\left(b_{2}+\alpha+2 d\right)}{d(d+\delta)} \\
& \triangleq-m_{1}\left(S-S^{*}\right)^{2}-m_{2}\left(E-E^{*}\right)^{2}-m_{3}\left(Q-Q^{*}\right)^{2}-m_{4}\left(R-R^{*}\right)^{2}-m_{5}\left(R-R^{*}\right)^{2}+\Lambda,
\end{aligned}
$$

where $\chi=(A / \beta(d+\delta))\left(b_{2}+\alpha+2 d\right), \quad m_{i}(i=1,2,3,4,5)$, and $\Lambda$ are defined in Theorem 5 . 
Integrating (39) from 0 to $t$ and taking the expectation on both sides we have

$$
\begin{aligned}
0 & \leq E V \leq E V(S(0), E(0), Q(0), I(0), R(0)) \\
& +E \int_{0}^{t}\left[-m_{1}\left(S-S^{*}\right)^{2}-m_{2}\left(E-E^{*}\right)^{2}-m_{3}\left(Q-Q^{*}\right)^{2}-m_{4}\left(I-I^{*}\right)^{2}-m_{5}\left(R-R^{*}\right)^{2}+\Lambda\right] \mathrm{d} u .
\end{aligned}
$$

Then, letting $M=\min \left\{m_{1}, m_{2}, m_{3}, m_{4}, m_{5}\right\}$, dividing $t$ on both sides of (44), and taking the superior limit, we get

$$
\underset{t \rightarrow \infty}{\limsup } \frac{1}{t} E \int_{0}^{t}\left[\left(S-S^{*}\right)^{2}+\left(E-E^{*}\right)^{2}+\left(Q-Q^{*}\right)^{2}+\left(I-I^{*}\right)^{2}+\left(R-R^{*}\right)^{2}\right] \mathrm{d} u \leq \frac{\Lambda}{M} \text {. }
$$

\section{Application of the Model}

5.1. Numerical Simulations. The numerical simulations are divided into two aspects. We first set values of the parameters to verify the theorem results obtained in this study. Specifically, we set $A=50, \beta=0.8, b_{1}=0.3, b_{2}=0.5, \alpha=0.3, c=0.2$, $\eta=0.18, d=0.2$, and $\delta=0.25$. Using different initial values for $(S(t), E(t), Q(t), I(t), R(t))$ such as $(1500,100,50,10,0)$, $(1000,200,60,20,0)$, and $(500,10,5,1,0)$, we numerically solve system (1). Figure 1 shows that when $R_{0}<1$, the epidemic will go to extinction, and the solutions with different initial values will converge to the disease-free equilibrium expressed as $E_{0}=(250,0,0,0,0)$, which coincides with the conclusion of Theorem 1. For $\beta=1.2$ and the other parameters set as before, it is verified that when $R_{0}>1$, the epidemic will continue to spread, and the solutions will converge to the endemic equilibrium expressed as $E_{*}=(199.1,12.95,9.248,8.82,8.2)$ in Figure 1.

If we set $\beta=1.1$ and $\sigma=0.8$, the other parameters remain unchanged, and the initial value is given by $(500,10,5,1,0)$, while these parameters satisfy $\beta^{2} / 2 \sigma^{2}=0.945<b_{2}+\alpha+d=1$, the condition of Theorem 4 is satisfied as shown in Figure 2, and in this case, the epidemic will die out. When $\beta=1.1, \sigma=0.01$, and $\sigma=0.04$, the other parameters and initial value are the same as before, and we obtain the asymptotic property of system (2) and the solutions with stochastic oscillation near the endemic equilibrium of system (1), as shown in Figure 3.

5.2. Case Study. In this section, we present some cases in which the proposed research model can be applied. After the WHO reported the first case on December 31, 2019, the COVID-19 pandemic has spread rapidly worldwide. To date, 220 countries have suffered from this pandemic. By May 30, 2021 , there were $171,474,925$ cases of global confirmed cases, and the cumulative mortality reached $3,565,330$ cases. The infectious, noxious, and transmission speeds of the epidemic are shocking. Some countries are still trapped in the epidemic; here, we analyze data from France, Italy, and the UK. The data used in our study were extracted from Worldometers [29], an online freely available repository. In this study, we consider 95 days of time-series data of currently infected patients from three countries from February 24 to May 30, 2021. The source of the parameters is as follows. The population of Italy [30] is approximately 60,500,000, which we set it as the initial value of the total population. The value of $A$ can be set on the basis of the number of births in Italy, 1427. The average life expectancy in Italy is 84.01 [31]. Given that $1 / d$ is the average life expectancy, the value of $d$ can be calculated as $d=1 /(84.01 \times 365) \approx 3.2612 \times 10^{-5}$. Through the same calculations, we derive some parameters for France and the UK. We also set the range of $\alpha$, which represents the rate of exposure to infection according to [32]. Because the average recovery period in France is approximately 13.6 days [33], the parameter $\eta$ is set to $1 / 13.6 \approx 0.0735$. According to [34], we take the average recovery period in Italy as 13.15 days; thus, in this case, we set the parameter $\eta=1 / 13.15 \approx 0.076$. The recovery rate of the $\mathrm{UK}, \beta, b_{1}, b_{2}$, and $c$ are estimated by the least square method.

We fit the parameters of the system by using the daily active COVID-19 cases in France, Italy, and the UK. The parameters resulting from fitting the actual active cases are shown in Table 1; the results are shown in Figure 4. We consider hospitalized infected patients as active cases in this part. Note from Figure 4 that the simulations are correct, and the epidemic development of these three countries shows a decreasing trend. It is evident that the people infection will not stop shortly. The government should take measures to reduce the number of newly infected people and maintain a decreasing trend.

According to the previous analysis, stochastic perturbation can change the solution of the system and the stage of 

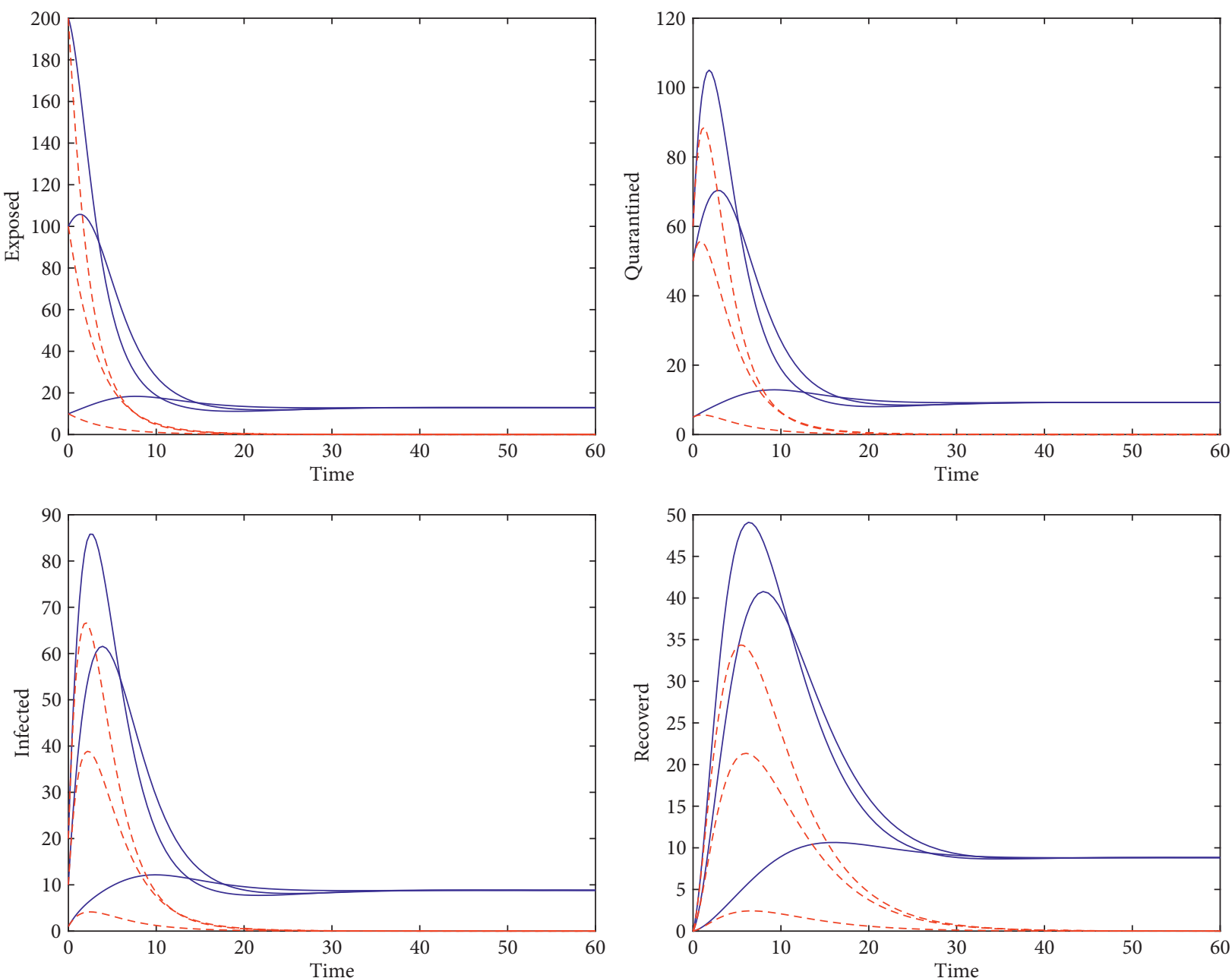

FIgURE 1: The local asymptotic stability of the equilibria of system (1). The blue line is the case of $R_{0}>1$, and the red dash line is the case of $R_{0}<1$.

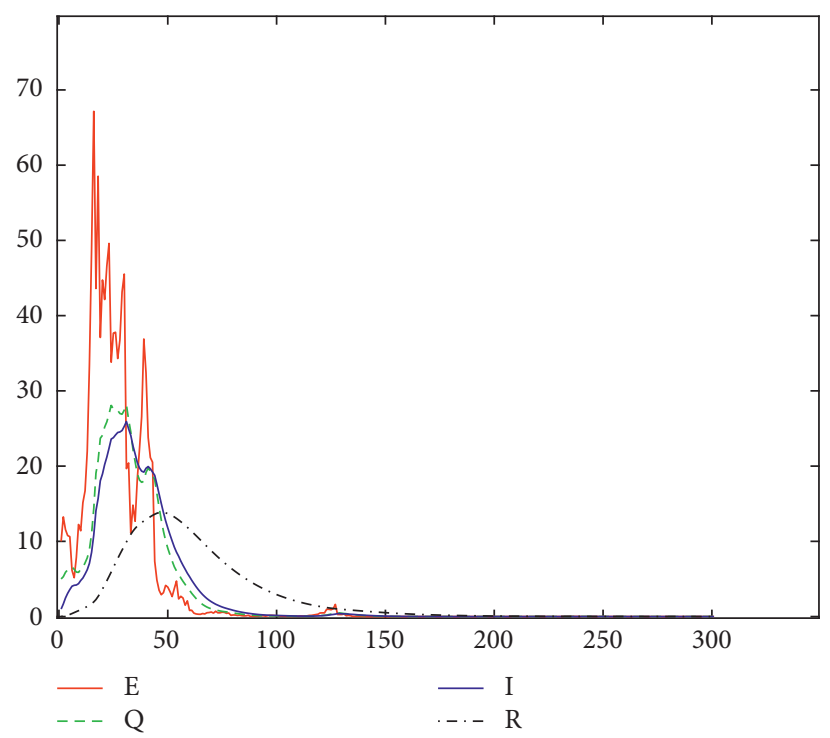

Figure 2: The epidemic extinction with stochastic perturbation. 

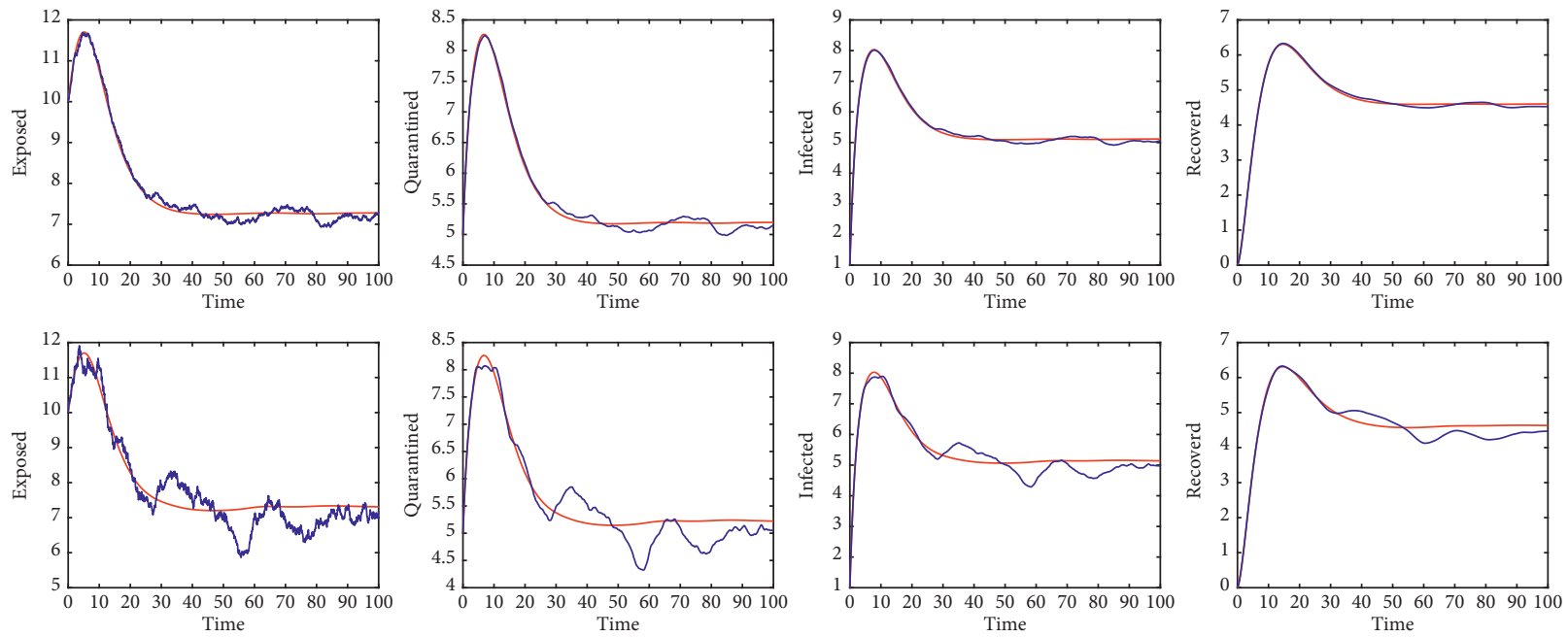

Figure 3: The asymptotic solutions of the deterministic and stochastic system. The first row is the case of $\sigma=0.01$, and the second row is the case of $\sigma=0.04$.

TABLE 1: Description and values of parameters.

\begin{tabular}{|c|c|c|c|c|c|}
\hline Parameter & Definition & France & Italy & The UK & Source \\
\hline$A$ & Total input & 2218 & 1427 & 2356 & {$[30]$} \\
\hline$\beta$ & Disease transmission rate & 0.926 & 0.571 & 0.999 & Estimated \\
\hline$d$ & Natural death rate & $3.3616 \times 10^{-5}$ & $3.2612 \times 10^{-5}$ & $3.2929 \times 10^{-5}$ & {$[31]$} \\
\hline$\eta$ & The recovery rate of $I$ & 0.0735 & 0.076 & 0.0714 & {$[33,34]$,estimated } \\
\hline$b_{1}$ & The rate that $Q$ becomes $S$ & 0.1 & 0.012 & 0.1 & Estimated \\
\hline$b_{2}$ & The rate that $E$ becomes $Q$ & 0.718 & 0.3354 & 0.7734 & Estimated \\
\hline$\alpha$ & The rate that $E$ becomes $I$ & 0.1923 & 0.1924 & 0.199 & [32] \\
\hline$c$ & The rate that $Q$ becomes $I$ & 0.6308 & 0.8 & 0.1 & Estimated \\
\hline$\delta$ & The mortality rate of $I$ & 0.0249 & 0.0338 & 0.0294 & [31] \\
\hline
\end{tabular}

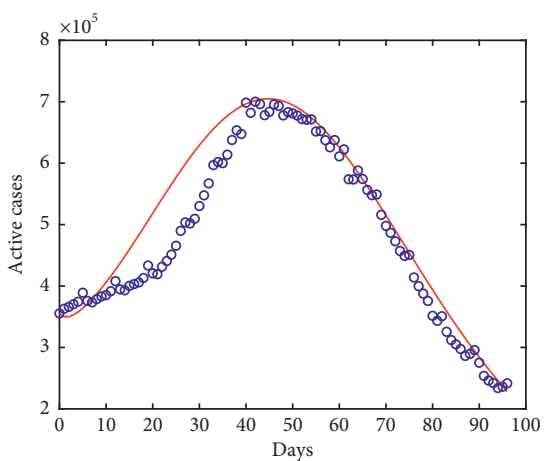

(a)

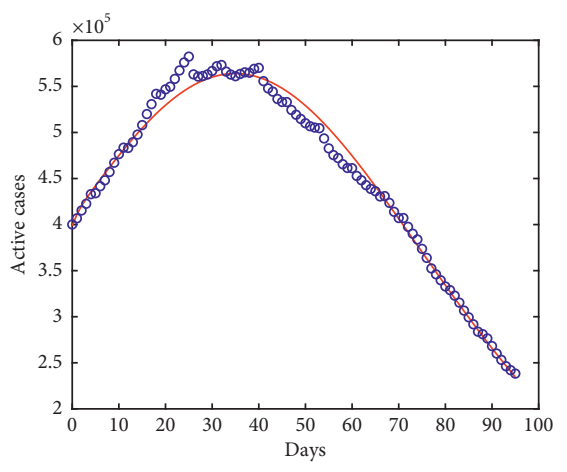

(b)

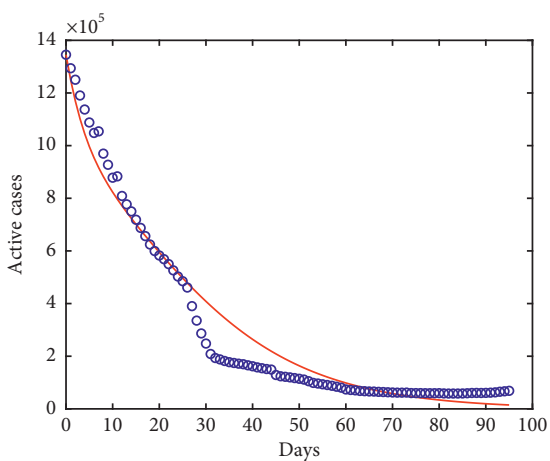

(c)

Figure 4: The fitting results of system (1). (a) France. (b) Italy. (c) The UK.

disease development. The epidemic becomes extinct when the noise intensity is sufficiently large. However, if the noise intensity is small, the overall trend of disease development will not change. This situation can be seen in Figure 5, which shows the solutions of the deterministic and stochastic models with $\sigma=0.02$, illustrating that the stochastic solution fluctuates around the deterministic solution. This leads to conclude that the government should take some measures to 

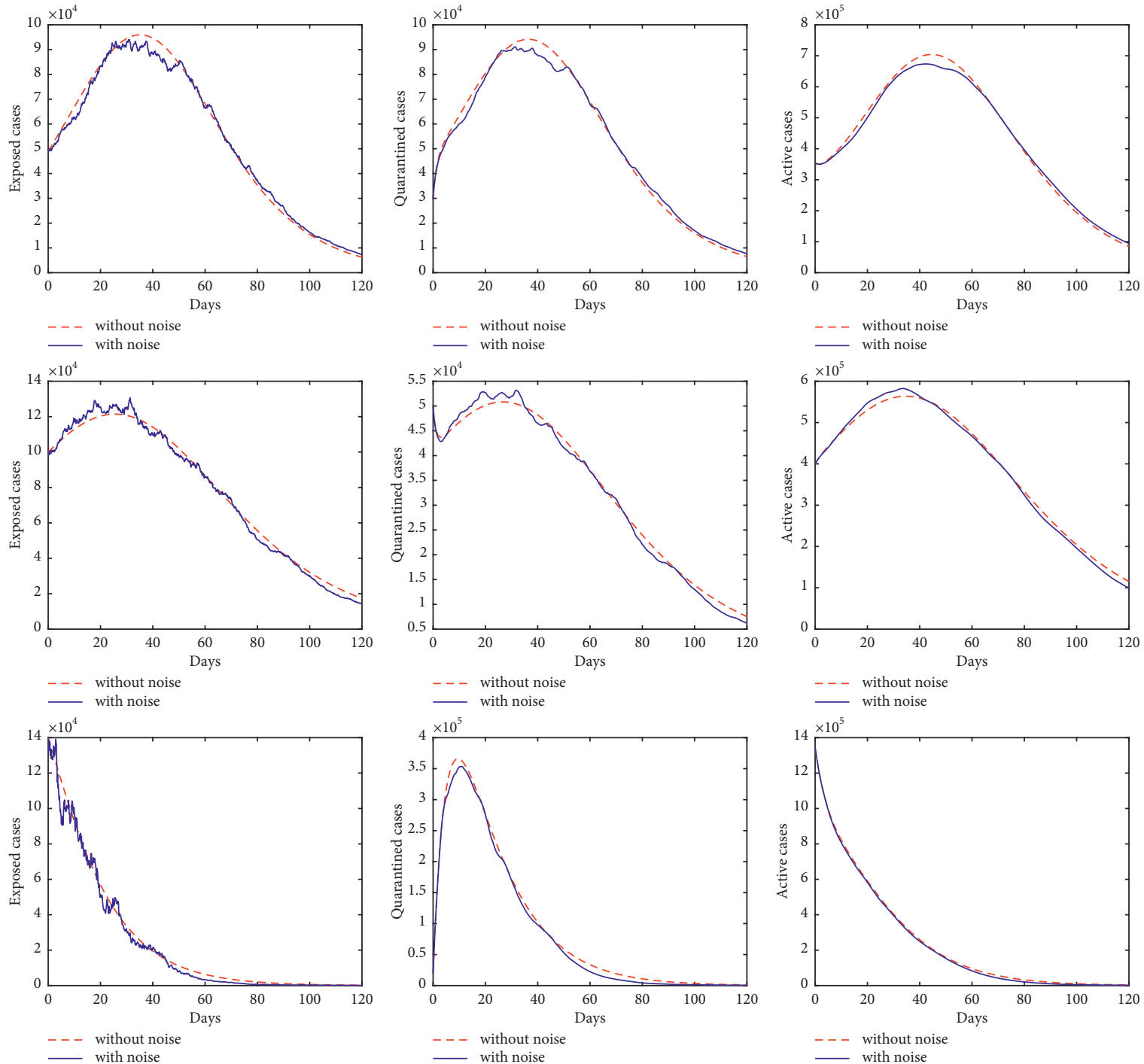

Figure 5: The solutions of system (2) with $\sigma=0.02$. The first row is the case of France, the middle row is the case of Italy, and the last row is the case of the UK. All parameters are listed in Table 1.

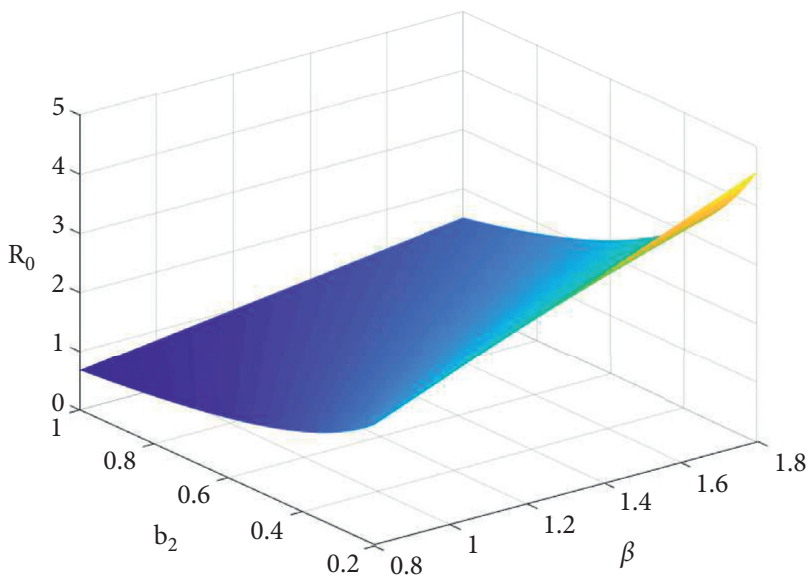

(a)

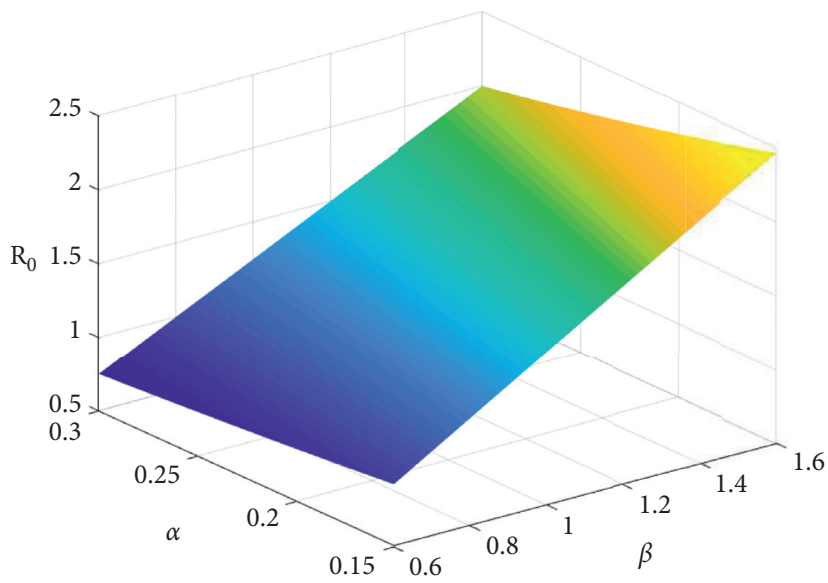

(b)

Figure 6: The relationship between parameters and $R_{0}$. (a) $\beta, b_{2}$, and $R_{0}$. (b) $\beta$, $\alpha$, and $R_{0}$. 
prevent new infections. Figure 6 shows the increase in $b_{2}$, which represents the strengthening isolation, and $\alpha$, which means that raising nucleic acid detection will effectively reduce the risk of disease transmission.

\section{Conclusions}

In 2020, new coronavirus pneumonia seriously affected people's daily life and hindered social development. More than 200 countries worldwide suffered from this epidemic. Prevention of this coronavirus pneumonia has become the most important issue worldwide. Infectious disease models constitute an effective tool for analyzing and understanding the trend of disease spread; therefore, we built a mathematical model with standard incidence based on the spread of COVID-19. We obtained the basic reproduction number, which affects the spread of the disease, and proved that when $R_{0}<1$, the disease-free equilibrium is locally asymptotically stable, and when $R_{0}>1$, the endemic equilibrium is locally asymptotically stable. Then, we incorporated stochastic perturbation in the deterministic model because there exists stochastic influence in the process of disease spread. Specifically, we obtained the conditions under which the epidemic goes to extinction and the asymptotic property near the endemic equilibrium of the deterministic model.

Finally, we verified the results in two aspects. First, we set some parameters to verify the proposed theorem. Second, we fitted the active data cases using the least square method. In Figure 4, we effectively fitted the active cases in France, Italy, and the UK. The active cases of these three countries all decreased. In the first part of the numerical simulations, we took into account that adding a stochastic disturbance will prevent the spread of the disease. Therefore, we considered stochastic disturbance in the model for comparison with the deterministic model. The parameters in Table 1 do not satisfy the condition of disease extinction, so the epidemic will not go to extinction. It is well known that white noise with sufficiently large intensity can lead to the extinction of the disease. Hence, we can control the development of the disease by increasing the noise intensity.

Random perturbance can be considered as a humanissue disturbance. Therefore, governments must take measures to decrease the number of infections, e.g., population lockdown, increase of media publicity, rational allocation of medical resources, and appeal to the public to pay attention to personal hygiene. Some suggestions, such as increasing the isolation and raising nucleic acid detection, should be made. With these suggestions, the number of infections will be reduced. Currently, novel coronavirus pneumonia vaccines have been developed. It is believed that a reasonable combination of government control and vaccination strategies will eliminate the epidemic. Next, we will also consider the model with immunity to analyze the impact of immune intensity on the spread of infectious diseases.

\section{Data Availability}

The data used to support the findings of this study are available from the corresponding author upon request.

\section{Conflicts of Interest}

The authors declare that they have no conflicts of interest.

\section{Acknowledgments}

The authors would like to express their heartfelt appreciation for the financial support provided by the Research Project supported by the National Nature Science Foundation of China (nos. 12071445 and 12001501), Fund for Shanxi (1331KIRT), and the Outstanding Youth Fund of North University of China.

\section{References}

[1] World health organization, https://www.who.int/ westernpacific/emergencies/COVID-19.

[2] F. C. Jasper, S. H. Yuan, K. H. Kok, and K. Y. Yuen, "A familial cluster of pneumonia associated with the 2019 novel coronavirus indicating person-to-person transmission: a study of a family cluster," The Lancet, vol. 395, pp. 514-523, 2020.

[3] C. Rothe, M. Schunk, P. Sothmann et al., "Transmission of 2019-nCoV infection from an asymptomatic contact in Germany," New England Journal of Medicine, vol. 382, no. 10, pp. 970-971, 2020.

[4] S. Lai, N. W. Ruktanonchai, L. Zhou et al., "Effect of nonpharmaceutical interventions to contain COVID-19 in China," Nature, vol. 585, no. 7825, pp. 410-413, 2020.

[5] H. Lau, V. Khosrawipour, P. Kocbach et al., "The positive impact of lockdown in Wuhan on containing the COVID-19 outbreak in China," Journal of Travel Medicine, vol. 27, pp. 1-7, 2020.

[6] K. Prem, Y. Liu, T. W. Russell et al., "The effect of control strategies to reduce social mixing on outcomes of the COVID19 epidemic in Wuhan, China: a modelling study," The Lancet. Public health, vol. 5, pp. E261-E270, 2020.

[7] N. C. Grassly and C. Fraser, "Mathematical models of infectious disease transmission," Nature Reviews Microbiology, vol. 6, no. 6, pp. 477-487, 2008.

[8] Y. Zhou, W. Zhang, and S. Yuan, "Survival and stationary distribution of a SIR epidemic model with stochastic perturbations," Applied Mathematics and Computation, vol. 244, pp. 118-131, 2014.

[9] S. Shivram, V. H. Badshah, and V. Gupta, "Analysis of an SEIR epidemic model with a saturated incidence rate," Asian Journal of Mathematics and Computer Research, vol. 19, pp. 124-138, 2017.

[10] Z. W. Cao, W. Feng, X. D. Wen, L. Zu, and M. Cheng, "Dynamics of a stochastic SIQR epidemic model with standard incidence," Physica A, vol. 527, Article ID 121180, 2019.

[11] G.-Q. Sun, M.-T. Li, J. Zhang, W. Zhang, X. Pei, and Z. Jin, "Transmission dynamics of brucellosis: mathematical modelling and applications in China," Computational and Structural Biotechnology Journal, vol. 18, pp. 3843-3860, 2020.

[12] G.-Q. Sun, H.-T. Zhang, J.-S. Wang et al., "Mathematical modeling and mechanisms of pattern formation in ecological systems: a review," Nonlinear Dynamics, vol. 104, no. 2, pp. 1677-1696, 2021.

[13] B. Tang, X. Wang, Q. Li et al., "Estimation of the transmission risk of the 2019-nCoV and its implication for public health interventions," Journal of Clinical Medicine, vol. 9, Article ID 462, 2020.

[14] D. Adam, "Special report: the simulations driving the world's response to COVID-19," Nature, vol. 580, no. 7803, pp. 316-318, 2020. 
[15] A. J. Kucharski, T. W. Russell, C. Diamond et al., "Early dynamics of transmission and control of COVID-19: a mathematical modelling study," The Lancet Infectious Diseases, vol. 20, no. 5, pp. 553-558, 2020.

[16] F. Ndariou, I. Area, J. Nieto, and D. F. Torres, "Mathematical modeling of COVID-19 transmission dynamics with a case study of Wuhan," Chaos, Solitons \& Fractals, vol. 135, Article ID 109846, 2020.

[17] R. MHDM, R. G. Silva, V. C. Mariani, and L. S. Coelho, "Short-term forecasting COVID-19 cumulative confirmed cases: perspectives for Brazil," Chaos, Solitons \& Fractals, vol. 135, Article ID 109853, 2020.

[18] D. Adak, A. Majumder, and N. Bairagi, "Mathematical perspective of COVID-19 pandemic: disease extinction criteria in deterministic and stochastic models," Chaos, Solitons \& Fractals, vol. 142, Article ID 110381, 2020.

[19] M. Mandal, S. Jana, S. K. Nandi, A. Khatua, S. Adak, and T. K. Kar, "A model based study on the dynamics of COVID19: prediction and control," Chaos, Solitons, and Fractals, vol. 136, Article ID 109889, 2020.

[20] S. Khajanchi and K. Sarkar, "Forecasting the daily and cumulative number of cases for the COVID-19 pandemic in India," Chaos, vol. 30, Article ID 071101, 2020.

[21] G.-Q. Sun, S.-F. Wang, M.-T. Li et al., "Transmission dynamics of COVID-19 in Wuhan, China: effects of lockdown and medical resources," Nonlinear Dynamics, vol. 101, no. 3, pp. 1981-1993, 2020.

[22] C. F. Manski and F. Molinari, "Estimating the COVID-19 infection rate: anatomy of an inference problem," Journal of Econometrics, vol. 220, no. 1, pp. 181-192, 2021.

[23] K. Karako, P. Song, Y. Chen, and W. Tang, "Analysis of COVID-19 infection spread in Japan based on stochastic transition model," Bioscience Trends, vol. 14, no. 2, pp. 134-138, 2020.

[24] D. Anwarud, A. Khan, and D. Baleanu, "Stationary distribution and extinction of stochastic coronavirus (COVID-19) epidemic model," Chaos, Solitons \& Fractals, vol. 139, Article ID 110036, 2020.

[25] B. Brahim, C. Toms, E. F. Mohamed, and E. K. Mohamed, "Dynamics of a stochastic coronavirus (COVID-19) epidemic model with Markovian switching," Chaos, Solitons \& Fractals, vol. 141, Article ID 110361, 2020.

[26] M. A. Akinlar, M. Inc, J. F. Gómez-Aguilar, and B Boutarfa, "Solutions of a disease model with fractional white noise," Chaos, Solitons, and Fractals, vol. 137, Article ID 109840, 2020.

[27] P. van den Driessche and J. Watmough, "Reproduction numbers and sub-threshold endemic equilibria for compartmental models of disease transmission," Mathematical Biosciences, vol. 180, no. 1-2, pp. 29-48, 2002.

[28] X. R. Mao, Stochastic Differential Equations and Applications, Horwood Publishing, Chichester, UK, 1997.

[29] Worldometer, https://www.worldometers.info/coronavirus.

[30] Live births, https://countrymeters.info/en.

[31] Life expectancy, https://www.worldometers.info/population.

[32] S. A. Lauer, K. H. Grantz, Q. Bi et al., "The incubation period of coronavirus disease 2019 (COVID-19) from publicly reported confirmed cases: estimation and application," Annals of Internal Medicine, vol. 172, no. 9, pp. 577-582, 2020.

[33] R. Forien, G. Pang, and É. Pardoux, "Estimating the state of the COVID-19 epidemic in France using a model with memory," Royal Society Open Science, vol. 8, no. 3, Article ID 202327, 2021.
[34] G. Marino, B. Enrico, M. Lorenzo et al., "Spread and dynamics of the COVID-19 epidemic in Italy: effects of emergency containment measures," Proceedings of the National Academy of Sciences, vol. 117, pp. 10484-10491, 2020. 